\title{
Effectiveness of telerehabilitation on short-term quality of life of patients after esophageal cancer surgery during COVID-19: a single-center, randomized, controlled study
}

\author{
Keqing Chen ${ }^{1 \#}$, Fei Yao ${ }^{2 \#}$, Xiaoyu Chen ${ }^{3}$, Yanjuan Lin ${ }^{4,5}$, Minqiang Kang ${ }^{1}$ \\ ${ }^{1}$ Department of Thoracic Surgery, Union Hospital, Fujian Medical University, Fuzhou, China; ${ }^{2}$ Department of endocrinology, Fuzhou Hospital of \\ Traditional Chinese Medicine, Fuzhou, China; ${ }^{3}$ Department of Thoracic Surgery, the First Affiliated Hospital of Fujian Medical University, Fuzhou, \\ China; ${ }^{4}$ Department of Nursing, Union Hospital, Fujian Medical University, Fuzhou, China; ${ }^{5}$ Department of Cardiac Surgery, Union Hospital, \\ Fujian Medical University, Fuzhou, China \\ Contributions: (I) Conception and design: Y Lin, M Kang; (II) Administrative support: None; (III) Provision of study materials or patients: None; \\ (IV) Collection and assembly of data: K Chen, F Yao, K Chen, X Chen; (V) Data analysis and interpretation: K Chen, F Yao, K Chen, X Chen; (VI) \\ Manuscript writing: All authors; (VII) Final approval of manuscript: All authors. \\ \#These authors contributed equally to this work. \\ Correspondence to: Yanjuan Lin. Department of Nursing, Union Hospital, Fujian Medical University, No. 29 Xinquan Road, Fuzhou 350001, China. \\ Email: fjxhyj@163.com; Minqiang Kang. Department of Thoracic Surgery, Union Hospital, Fujian Medical University, No. 29 Xinquan Road, \\ Fuzhou 350001, China. Email: Kangmingqiang@sina163.com.
}

Background: The occurrence of postoperative complications may lead to delayed recovery and a decline in physical function in the first 3 months after esophagectomy. The outbreak of COVID-19 imposed physical and emotional obstacles for traditional face-to-face rehabilitation. Meanwhile, the effectiveness of telerehabilitation remained unknown. In this study, we aimed to investigate the effectiveness of telerehabilitation.

Methods: A cohort of 86 patients who received minimally invasive esophagectomy between September 2020 and January 2021 was randomly allocated into two groups. The telerehabilitation group received additional online consulting and training, including (I) precautions for nutritional support; (II) swallowing function training; (III) respiratory function training; (IV) guidance and feedback on matters such as patient's current vital signs, wound status, medication, and sleep status. The primary outcome was the change of quality of life (QOL) of each patient at 3 months after surgery.

Results: No serious adverse events were observed in either group. The telerehabilitation group showed significant improvements in pain using the OLQ-C30 scale $(\mathrm{P}<0.001)$, and in choking using the QLQOES18 scale $(\mathrm{P}<0.001)$. The comparison of the QLQ-C30 and QES-18 score changes at three months after discharge revealed that nearly all aspects in the telerehabilitation group displayed more score changes with significant changes in the appetite loss and pain part $(\mathrm{P}<0.001$ and $\mathrm{P}<0.05$, respectively). The score changes in QLQ-OES18 revealed significant improvement in swallowing saliva $(\mathrm{P}<0.05)$, as well slight improvements in choking, dry mouth, taste, and cough without significance.

Conclusions: Our study demonstrated that telerehabilitation was at least an important supplement to traditional face-to-face consulting and training for patients after esophageal cancer surgery during the COVID-19 period.

Trial Registration: Chinese Clinical Trial Registry ChiCTR2100049186.

Keywords: Telerehabilitation; minimally invasive esophagectomy; quality of life (QOL); COVID-19

Submitted Jun 08, 2021. Accepted for publication Aug 16, 2021.

doi: 10.21037/jgo-21-385

View this article at: https://dx.doi.org/10.21037/jgo-21-385 


\section{Introduction}

Esophageal cancer is the sixth leading cause of cancer death globally (1). While the primary curative treatment for patients is surgical resection of the esophagus (2), the occurrence of postoperative complications may lead to a delayed postoperative recovery and an obvious decline in physical function in the first 3 months after surgery (3). Esophagectomy patients often complain of fatigue, appetite loss, sleep disturbance, trouble with swallowing saliva, and choking, which may result from altered cardiopulmonary function, generalized muscle weakness, and malnutrition (4). Multiple prior studies have demonstrated that better postoperative rehabilitation was associated with reduced postoperative complications and improved the quality of life (QOL) for patients after esophageal cancer surgery (4-6). Traditionally, patients receive face-to-face rehabilitation training, especially during the first three months after surgery. Unfortunately, the outbreak of COVID-19 posed physical and emotional obstacles for patients returning to hospitals after surgery, which sometimes made face-to-face rehabilitation consulting and training impossible.

A telerehabilitation service refers to rehabilitation service provided through electronic multi-media communication technologies (7). As telerehabilitation consulting and training can be conducted in the patient's home, it may overcome the inconvenience and risk of infection in a crowded environment. Till now, there's a lack of evidence evaluating the effectiveness of telerehabilitation in patients with esophageal cancer. One prospective feasibility study including 22 patients who underwent esophagectomy has demonstrated that a postoperative physiotherapeutic intervention with telerehabilitation is feasible for patients with postoperative complications (8). Other studies were either the report involving patient satisfaction with virtual visits during COVID-19 or the evaluation of the usefulness of online stroke $(7,9)$ and cardiac rehabilitation (10).

To investigate the effectiveness of telerehabilitation consulting and training, a cohort of 86 patients who received minimally invasive esophagectomy between September 2020 and January 2021 was randomly divided into two groups. One group received common consulting whenever they returned to the thoracic surgery clinic, and the other received regular telerehabilitation consulting and training. Herein, we report our observations in this cohort. We present the following article in accordance with the CONSORT reporting checklist (available at https://dx.doi. org/10.21037/jgo-21-385).

\section{Methods}

\section{Study cobort, randomization, and ethics approvement}

A cohort of 86 patients who received minimally invasive esophagectomy for pathologically diagnosed esophageal cancer between September 2020 and January 2021 were recruited from the surgical wards of the Department of Thoracic Surgery, Fujian Medical University Union Hospital. The inclusion criteria were: age ranging from 18 years old to 75 years old; patients who had undergone minimally invasive esophagectomy; patients who were discharged within 30 days of surgery; the estimated survival time was over 90 days. Exclusion criteria for both telerehabilitation and control groups included a diagnosis of severe cardiopulmonary diseases before surgery, a diagnosis of severe postoperative complications such as postoperative chylothorax or anastomotic fistula, a need for professional medical equipment which was provided only at hospital, or refusal to participate in follow-up assessments. Eventually, 80 patients who received minimally invasive esophagectomy agreed to participate and signed written informed consents. The trial design type was two-parallel and a method of simple randomization was applied. Briefly, a series of random numbers were generated using the SPSS Software and assigned into two groups in a 1:1 ratio ahead of the first patient's admission in the study. The two groups were: (I) a telerehabilitation group who received additional telerehabilitation consulting and training; (II) a control group receiving standard consultation whenever they returned to the thoracic surgery clinic. Subsequently, patients who agreed to participate in the study were received a random number and assigned into the two groups at discharge. The following-up telerehabilitation intervention and clinical assessments were performed by different groups of researchers (Study flowchart in Figure S1). The study protocol was approved by the ethics committees of Fujian Medical University Union Hospital (No.: 2020KY0160). The study was conducted in accordance with the Declaration of Helsinki (as revised in 2013) and was registered on the Chinese Clinical Trial Registry (ChiCTR2 1000049186). The full trial protocol can be accessed on the Chinese Clinical Trial Registry website (https://www.chictr.org.cn/).

\section{Telerebabilitation intervention}

Telerehabilitation intervention was provided by a medical 
care group which included the head of the nursing department in thoracic surgery, at least one doctor who participated in the surgery, and three experienced nurses. Participants in the telerehabilitation group received a 3-month home-based telerehabilitation intervention after discharge, and a WeChat group including the medical care group, the patient, and the patient's family was created at discharge. The telerehabilitation intervention included the following: (I) precautions for nutritional support; (II) swallowing function training; (III) respiratory function training; (IV) guidance and feedback on matters such as patient's current vital signs, wound status, medication, and sleep status. The intervention was delivered using daily small tips, training videos, and regular online consulting. Patients, accompany by their care givers, were asked to receive training on schedule. The attendance rate on regular online consulting was also be followed.

\section{Clinical assessments and primary outcome}

The European Organization for Research and Treatment of Cancer Quality of Life (QOL) Questionnaire Core 30 (EORTC QLQ-C30) scale (version 3.0) (11), which contains scales and items addressing functional aspects of QOL and symptoms that commonly occur in patients with cancer, and its esophageal cancer supplementary scale EORTC QLQ-OES18 (12) were used for the assessments of QOL. Assessments were performed face-to-face at discharge and at 3 months post-operation by another group of trained researchers. Scores were calculated to the 100-point system in accordance with instructions in the EORTC QLQ-C30 scoring manual (13). The primary outcome was measured by the score changes at three months post-operation compared to the baseline at discharge.

\section{Statistical analysis}

Statistical analyses were performed using SPSS version 25.0 (SPSS Inc., Chicago, IL, USA). Quantitative measures were summarized with descriptive statistics, such as mean and $\mathrm{SD}$, and categorical data were expressed as $\mathrm{n}(\%)$. Chi-square tests were performed to determine if the two groups had the same distribution. The student's $t$-tests were performed to compare differences between two groups for measurement data. The normality of variables was tested using Kolmogorov-Smirnov test before the use of student's $t$-tests. Variables without normality were compared using
Mann-Whitney $\mathrm{U}$ tests. $\mathrm{P}$ values were two-tailed with a significance level of 0.05 .

\section{Results}

\section{Demographic and clinical characteristics of the study cohort}

The study flowchart and clinical follow-up schedule were displayed in Figure S1. The demographic data of participants is summarized in Table 1. Briefly, most were male, with an average age around $59.6 \pm 6.5$ and $59.8 \pm 7.0$ in the telerehabilitation and control groups, respectively. No differences were revealed in risk factors, including hypertension, diabetes, and smoking, and the dominant pathological pattern in both groups was squamous cell carcinoma, with one adenocarcinoma in the telerehabilitation group. The tumors in both groups were mostly located at the middle thoracic level, and most patients were in the early stages of disease, with no differences between the two groups.

\section{Baseline assessments at discharge}

Baseline assessments were performed at discharge for each participant, and as is shown in Table 2, there was no significant difference in the EORTC QLQ-C30 and QLQOES18 scores between groups.

\section{Assessments at 3 months post-operation}

The second face-to-face assessments were performed 3 months post-operation. No serious adverse events were observed in either group. As shown in Figure 1, both groups showed significantly increased scores in physical functions ( $\mathrm{P}<0.001$ for the telerehabilitation group and $\mathrm{P}<0.001$ for the control group) and social functions $(\mathrm{P}=0.002$ for the telerehabilitation group and $\mathrm{P}<0.001$ for the control group) 3 months post-operation. Both groups showed significantly decreased scores in fatigue $(\mathrm{P}<0.001$ for the telerehabilitation group and $\mathrm{P}<0.001$ for the control group) and nausea $(\mathrm{P}=0.003$ for the telerehabilitation group and $\mathrm{P}=0.01$ for the control group). However, it was noted that pain was significantly relieved only in the telerehabilitation group $(\mathrm{P}<0.001)$. Appetite loss in the control group was slightly but significantly increased $(\mathrm{P}=0.03)$, and the global QOLs in both groups improved significantly. Similar findings were revealed in the QLQ-OES18 scale with a much more significant improvement in the choking score in the telerehabilitation group $(\mathrm{P}<0.001)$. Horizontal 
Table 1 Demographic and clinical characteristics of the study

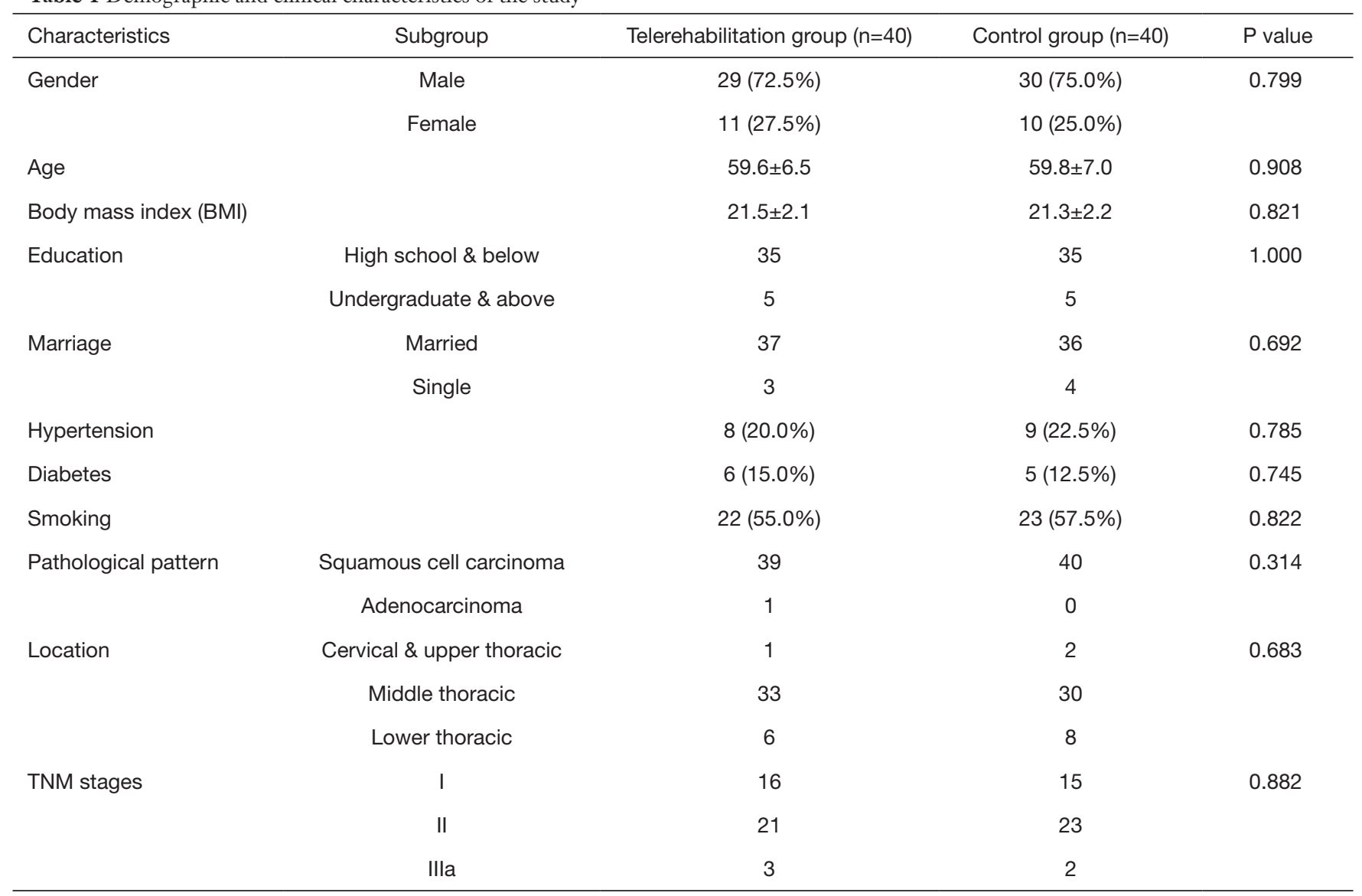

comparison at 3 months post-operation is displayed in Table 3. While there was no significant difference in function area between groups, the telerehabilitation group had much lower symptom scores in sleep disturbance, appetite loss, and financial impact $(\mathrm{P}=0.019, \mathrm{P}=0.005$, and $\mathrm{P}=0.041$, respectively). Moreover, the QLQ-OES18 scale revealed that the telerehabilitation group had much lower scores in swallowing saliva, choking, and cough $(\mathrm{P}=0.010$, $\mathrm{P}=0.024$, and $\mathrm{P}=0.041$, respectively).

The comparison of the QLQ-C30 and QES-18 score changes at three months after discharge was revealed in Figure 2. Similar to results in the horizontal comparison of the QLQ-C30 and QES18 score at 3 months postoperation, though there was no significant difference in function area between groups, more score changes were noticed in the emotional, cognitive, and role function part (Figure 2A). Moreover, nearly all aspects in the telerehabilitation group displayed more score changes with significant changes in the appetite loss and pain part
$(\mathrm{P}<0.001$ and $\mathrm{P}=0.04$, respectively, Figure $2 B)$. The score changes in QLQ-OES18 revealed significant improvement in swallowing saliva $(\mathrm{P}=0.02)$, as well slight improvements in choking, dry mouth, taste, and cough without significance (Figure 2C).

\section{Discussion}

Surgical resection of the esophagus is associated with multiple short-and long-term adverse effects, including high rates of postoperative complications, decreased muscle strength, altered cardiopulmonary function, emotional impact, and mortality. Whether preoperative functional training can benefit postoperative recovery after esophageal surgery is controversial $(14,15)$. Several studies have demonstrated that preoperative inspiratory training and nutrition optimizing improves perioperative functional capacity $(16,17)$. However, other studies queried the effectiveness of preoperative functional training (18-20), 
Table 2 QLQ-C30 and QLQ-OES18 scores at discharge between groups

\begin{tabular}{|c|c|c|c|c|}
\hline Scales and items & Telerehabilitation group $(n=40)$ & Control group $(n=40)$ & $\mathrm{t}$ value & $\mathrm{P}$ \\
\hline \multicolumn{5}{|l|}{ Function } \\
\hline Physical & $33.50 \pm 14.04$ & $32.67 \pm 14.62$ & -0.260 & 0.796 \\
\hline Role & $28.33 \pm 14.22$ & $26.25 \pm 16.83$ & -0.598 & 0.552 \\
\hline Cognitive & $56.67 \pm 16.80$ & $52.08 \pm 23.63$ & 1.593 & 0.115 \\
\hline Social & $51.25 \pm 20.46$ & $59.17 \pm 23.86$ & 0.243 & 0.809 \\
\hline Global quality of life & $27.45 \pm 12.07$ & $27.50 \pm 18.65$ & 0.014 & 0.989 \\
\hline \multicolumn{5}{|l|}{ Symptom scales } \\
\hline Pain & $75.83 \pm 15.54$ & $73.75 \pm 17.25$ & -0.568 & 0.572 \\
\hline Dyspnea & $59.17 \pm 15.99$ & $53.33 \pm 21.08$ & -1.394 & 0.167 \\
\hline Sleep disturbance & $48.33 \pm 18.41$ & $55.00 \pm 20.74$ & 1.520 & 0.132 \\
\hline Appetite loss & $65.00 \pm 7.36$ & $63.33 \pm 10.13$ & -0.842 & 0.402 \\
\hline Constipation & $55.83 \pm 20.52$ & $56.67 \pm 18.80$ & 0.189 & 0.850 \\
\hline Diarrhea & $47.50 \pm 22.50$ & $54.17 \pm 20.93$ & 1.372 & 0.174 \\
\hline Financial impact & $61.67 \pm 12.05$ & $60.83 \pm 12.05$ & -0.299 & 0.765 \\
\hline \multicolumn{5}{|l|}{ QLQ-OES18 } \\
\hline Choking & $53.33 \pm 24.81$ & $55.83 \pm 23.13$ & 0.466 & 0.642 \\
\hline Dry mouth & $67.50 \pm 21.99$ & $69.17 \pm 21.86$ & 0.340 & 0.735 \\
\hline Taste & $73.33 \pm 18.80$ & $71.67 \pm 20.74$ & -0.377 & 0.708 \\
\hline Cough & $75.83 \pm 19.95$ & $75.00 \pm 19.61$ & -0.188 & 0.851 \\
\hline Speech & $65.00 \pm 21.28$ & $65.83 \pm 23.25$ & 0.167 & 0.868 \\
\hline
\end{tabular}

which may make post-operation rehabilitation more important. Since the outbreak of COVID-19, the disease has spread rapidly with high contagiousness and mortality (21), and the risk of infection in attending hospitals has made many patients hesitate to return for face-to-face postoperation consulting and rehabilitation. Herein, we investigated the effectiveness of telerehabilitation consulting and training in a cohort of 86 patients who received minimally invasive esophagectomy between September 2020 and January 2021. Using EORTC QLQ-C30 and QLQ-OES18 scales, we found patients' QOL could be significantly improved at 3 months after surgery. However, additional telerehabilitation consulting and training may also help in pain-relief and solving problems in sleeping and 

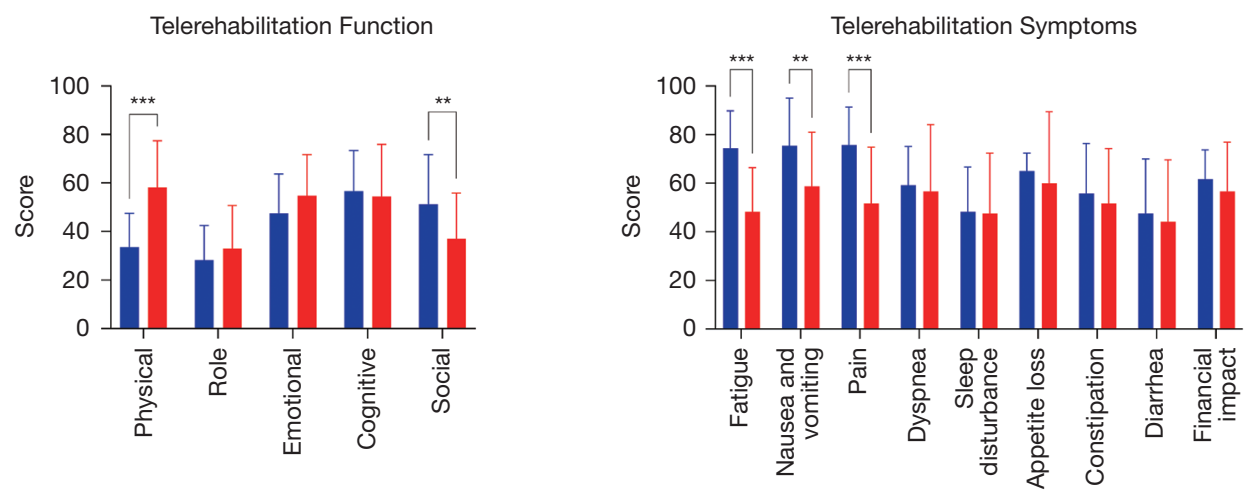

Telerehabilitation QLQ-OES18

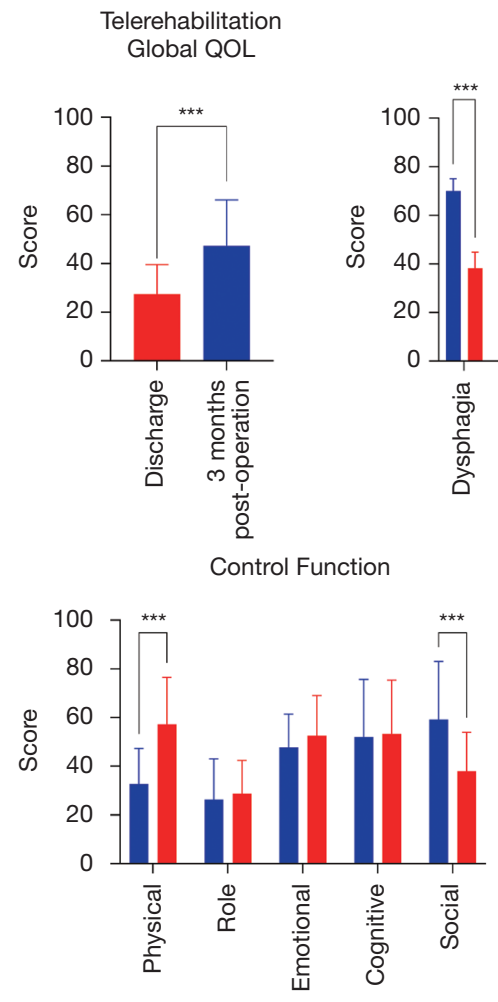

Control Function

Control Global QOL

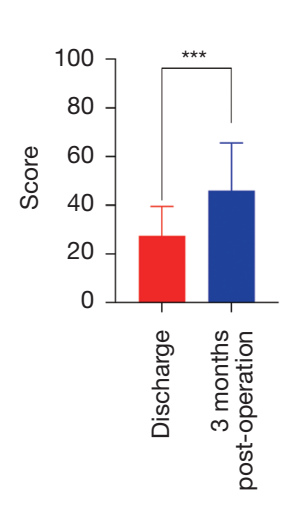

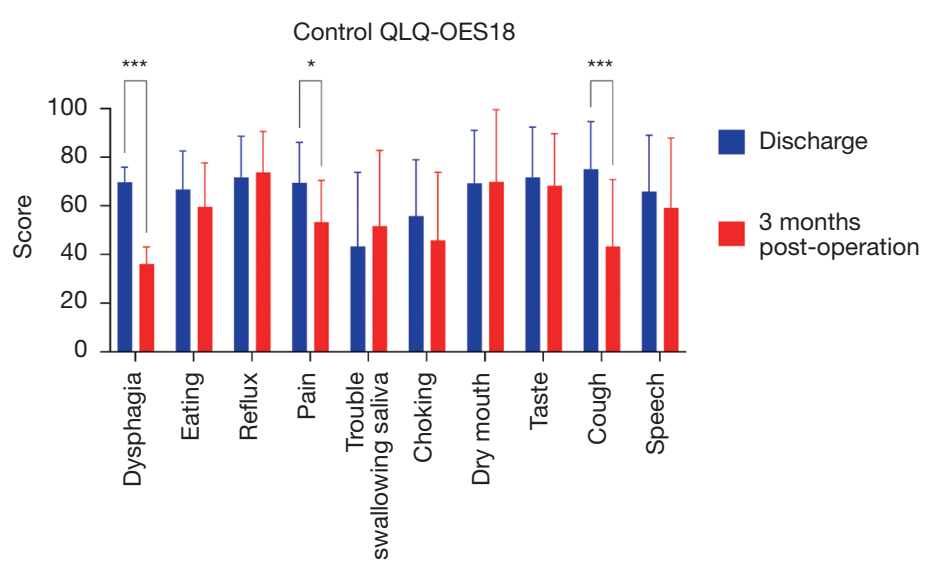

Figure 1 Comparison of QLQ-C30 and QLQ-OES18 scores between discharge and 3 months post-operation in the telerehabilitation group and the control group. *, $\mathrm{P}<0.05 ;{ }^{* *}, \mathrm{P}<0.01 ;{ }^{* * *}, \mathrm{P}<0.001$. 
Table 3 QLQ-C30 and QLQ-OES18 scores at 3 months after discharge between groups

\begin{tabular}{|c|c|c|c|c|}
\hline Scales and items & Telerehabilitation group $(n=40)$ & Control group $(n=40)$ & $\mathrm{t}$ value & $\mathrm{P}$ \\
\hline \multicolumn{5}{|l|}{ Function } \\
\hline Physical & $58.17 \pm 19.27$ & $57.17 \pm 19.39$ & -0.231 & 0.818 \\
\hline Role & $32.92 \pm 17.90$ & $28.75 \pm 13.60$ & -1.172 & 0.245 \\
\hline Cognitive & $54.58 \pm 21.35$ & $53.33 \pm 22.07$ & -0.257 & 0.797 \\
\hline Social & $37.08 \pm 17.08$ & $37.92 \pm 16.00$ & 0.225 & 0.822 \\
\hline Global quality of life & $47.29 \pm 18.81$ & $46.04 \pm 19.52$ & -0.292 & 0.771 \\
\hline \multicolumn{5}{|l|}{ Symptom scales } \\
\hline Pain & $51.67 \pm 23.20$ & $61.67 \pm 22.07$ & 1.975 & 0.052 \\
\hline Dyspnea & $56.67 \pm 27.43$ & $57.50 \pm 27.20$ & 0.136 & 0.892 \\
\hline Sleep disturbance & $47.50 \pm 24.91$ & $61.67 \pm 27.79$ & 2.401 & $0.019^{*}$ \\
\hline Appetite loss & $60.00 \pm 29.43$ & $76.67 \pm 21.62$ & 2.887 & $0.005^{\star}$ \\
\hline Constipation & $51.67 \pm 22.58$ & $60.00 \pm 22.90$ & 0.315 & 0.105 \\
\hline Diarrhea & $44.17 \pm 25.47$ & $54.17 \pm 20.93$ & 1.918 & 0.059 \\
\hline Financial impact & $56.67 \pm 20.26$ & $65.84 \pm 19.23$ & 2.076 & $0.041^{*}$ \\
\hline \multicolumn{5}{|l|}{ QLQ-OES18 } \\
\hline Choking & $31.67 \pm 27.92$ & $45.83 \pm 27.93$ & 2.300 & $0.024^{*}$ \\
\hline Dry mouth & $57.50 \pm 29.22$ & $69.83 \pm 29.71$ & 1.265 & 0.210 \\
\hline Taste & $64.17 \pm 24.32$ & $68.33 \pm 21.28$ & 0.815 & 0.417 \\
\hline Cough & $31.67 \pm 22.58$ & $43.33 \pm 27.43$ & 2.077 & $0.041^{*}$ \\
\hline Speech & $55.00 \pm 26.74$ & $59.17 \pm 28.73$ & 0.671 & 0.504 \\
\hline
\end{tabular}

*, $P<0.05$.

appetite loss.

The EORTC QLQ-C30 contains 30 items in total, including 15 areas: five functional areas (physical, role, emotion, cognition, and social function), one global QOL subscale, and nine symptom areas (pain, fatigue, nausea and vomiting, dyspnea, sleep disturbance, loss of appetite, constipation, diarrhea, and financial impact) (11). The OES18 is a supplementary symptom scale for esophageal cancer, which specifically includes 18 items such as dysphagia, eating, reflux, pain, saliva, choking, dry mouth, 

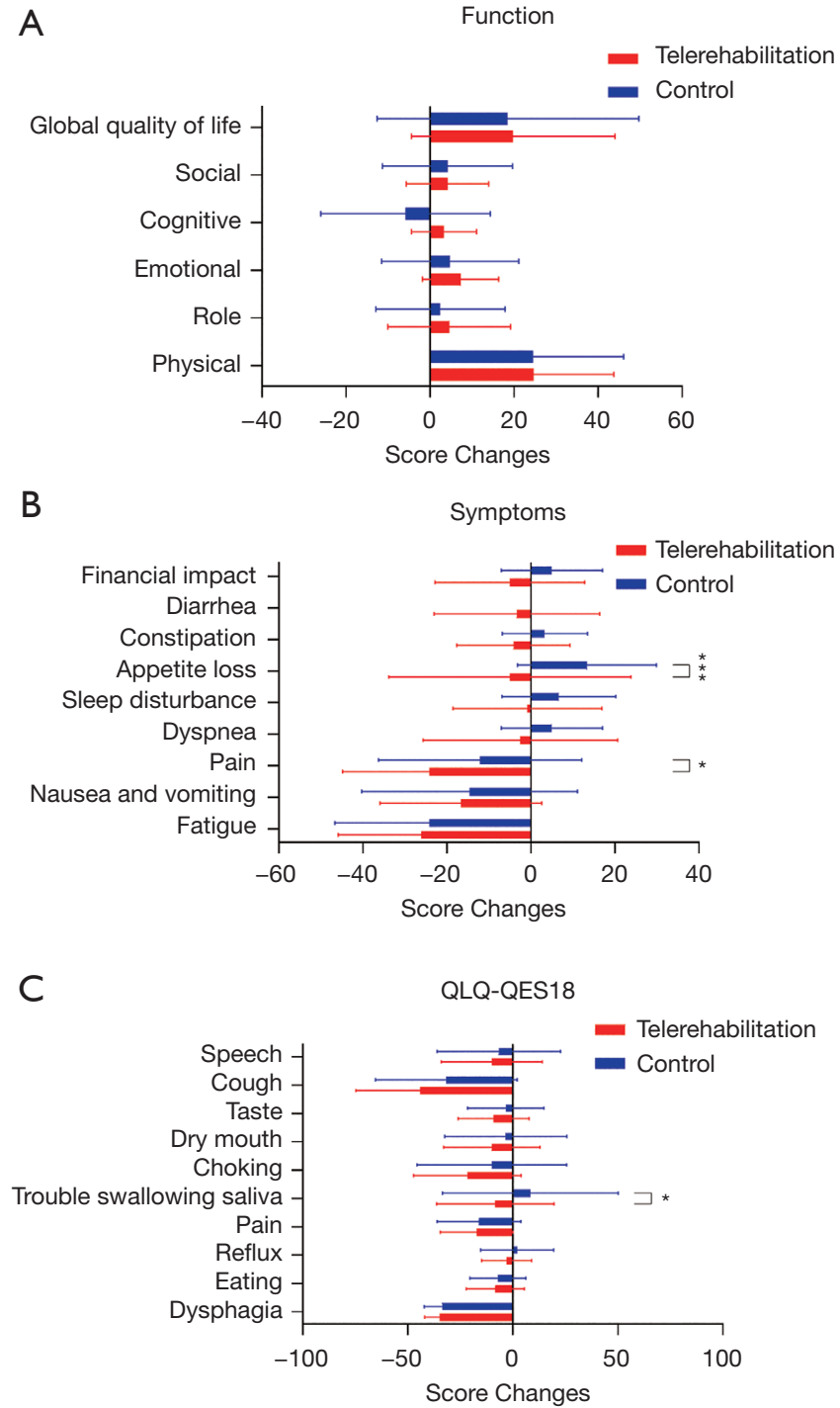

Figure 2 Comparison of the QLQ-C30 and QLQ-OES18 score changes at 3 months after discharge between the telerehabilitation group and the control group. * $\mathrm{P}<0.05$; ${ }^{* *}, \mathrm{P}<0.001$.

loss of appetite, cough, and speech (12). Many clinical trials, including trials conducted in a Chinese population, have proven that the combined application of QLQ-C30 and QLQ-OES18 can more accurately reflect the relationship between the symptoms of esophageal cancer patients and their QOL $(22,23)$.

In our study, the telerehabilitation group showed improvements mostly in pain relief, appetite loss and sleep disturbance. We assumed this was because telerehabilitation provided a platform for patients to share both their stories of discomfort and methods of coping, which could reduce their stress to some extent. This contrasts with the traditional method of rehabilitation, where patients must cope with their problems until returning to the clinic. Moreover, we found the patients in the telerehabilitation group coped with swallowing saliva better. Telerehabilitation can overcome the inconvenience of having to travel to the clinic and reduce the risk of exposure to coronavirus infection. The reduction in face-to-face consultations may also reduce costs associated with physical attendance to the clinic and allows more freedom for patients to return to work or engage in other activities. However, this study was conducted in a single institution with limited sample size, and the subjective scale was only evaluated at discharge and 3 months after operation. Multicenter studies with larger sample studies and longer follow-up durations are required to verify the results.

In conclusion, our study demonstrated that telerehabilitation was at least an important supplement to traditional face-toface consulting and training for patients after esophageal cancer surgery, especially during the COVID-19 period. However, not all areas in patients' QOL improved, which suggests a need for modifying the procedures and contents of telerehabilitation.

\section{Acknowledgments}

We would like to thank all participants for their cooperation in this study.

Funding: This work was supported by the Financial Special Project of Fujian Province (Grant number: 2020CZ011).

\section{Footnote}

Reporting Checklist: The authors have completed the CONSORT reporting checklist. Available at https://dx.doi. org/10.21037/jgo-21-385

Trial Protocol: Available at https://dx.doi.org/10.21037/jgo21-385

Data Sharing Statement: Available at https://dx.doi. org/10.21037/jgo-21-385

Conflicts of Interest: All authors have completed the ICMJE uniform disclosure form (available at https://dx.doi. org/10.21037/jgo-21-385). All authors reported that this 
work was supported by the Financial Special Project of Fujian Province (Grant number: 2020CZ011). The authors have no other conflicts of interest to declare.

Ethical Statement: The authors are accountable for all aspects of the work in ensuring that questions related to the accuracy or integrity of any part of the work are appropriately investigated and resolved. The study was conducted in accordance with the Declaration of Helsinki (as revised in 2013). The study protocol was approved by the ethics committees of Fujian Medical University Union Hospital (No.: 2020KY0160) and written informed consent was obtained from all patients.

Open Access Statement: This is an Open Access article distributed in accordance with the Creative Commons Attribution-NonCommercial-NoDerivs 4.0 International License (CC BY-NC-ND 4.0), which permits the noncommercial replication and distribution of the article with the strict proviso that no changes or edits are made and the original work is properly cited (including links to both the formal publication through the relevant DOI and the license). See: https://creativecommons.org/licenses/by-nc-nd/4.0/.

\section{References}

1. Siegel RL, Miller KD, Jemal A. Cancer statistics, 2016. CA Cancer J Clin 2016;66:7-30.

2. Grotenhuis BA, Wijnhoven BP, Grüne F, et al. Preoperative risk assessment and prevention of complications in patients with esophageal cancer. J Surg Oncol 2010;101:270-8.

3. O'Neill L, Moran J, Guinan EM, et al. Physical decline and its implications in the management of oesophageal and gastric cancer: a systematic review. J Cancer Surviv 2018;12:601-18.

4. Guinan EM, Dowds J, Donohoe C, et al. The physiotherapist and the esophageal cancer patient: from prehabilitation to rehabilitation. Dis Esophagus 2017;30:1-12.

5. Nakatsuchi T, Otani M, Osugi $H$, et al. The necessity of chest physical therapy for thoracoscopic oesophagectomy. J Int Med Res 2005;33:434-41.

6. Nakamura $M$, Iwahashi $M$, Nakamori M, et al. An analysis of the factors contributing to a reduction in the incidence of pulmonary complications following an esophagectomy for esophageal cancer. Langenbecks Arch Surg 2008;393:127-33.
7. Cramer SC, Dodakian L, Le V, et al. Efficacy of HomeBased Telerehabilitation vs In-Clinic Therapy for Adults After Stroke: A Randomized Clinical Trial. JAMA Neurol 2019;76:1079-87.

8. van Egmond MA, Engelbert RHH, Klinkenbijl JHG, et al. Physiotherapy With Telerehabilitation in Patients With Complicated Postoperative Recovery After Esophageal Cancer Surgery: Feasibility Study. J Med Internet Res 2020;22:e16056.

9. Scherrenberg M, Frederix I, De Sutter J, et al. Use of cardiac telerehabilitation during COVID-19 pandemic in Belgium. Acta Cardiol 2020. [Epub ahead of print]. doi: 10.1080/00015385.2020.1786625.

10. Chang MC, Boudier-Revéret M. Usefulness of Telerehabilitation for Stroke Patients During the COVID-19 Pandemic. Am J Phys Med Rehabil 2020;99:582.

11. Aaronson NK, Ahmedzai S, Bergman B, et al. The European Organization for Research and Treatment of Cancer QLQ-C30: a quality-of-life instrument for use in international clinical trials in oncology. J Natl Cancer Inst 1993;85:365-76.

12. Blazeby JM, Conroy T, Hammerlid E, et al. Clinical and psychometric validation of an EORTC questionnaire module, the EORTC QLQ-OES18, to assess quality of life in patients with oesophageal cancer. Eur J Cancer 2003;39:1384-94.

13. Fayers P, Aaronson N, Bjordal K, et al. EORTC QLQ-C30 Scoring Manual. 3rd ed. Brussels: European Organisation for Research and Treatment of Cancer, 2001.

14. Elliott JA, Doyle SL, Murphy CF, et al. Sarcopenia: Prevalence, and Impact on Operative and Oncologic Outcomes in the Multimodal Management of Locally Advanced Esophageal Cancer. Ann Surg 2017;266:822-30.

15. Gannon JA, Guinan EM, Doyle SL, et al. Reduced fitness and physical functioning are long-term sequelae after curative treatment for esophageal cancer: a matched control study. Dis Esophagus 2017;30:1-7.

16. Minnella EM, Awasthi R, Loiselle SE, et al. Effect of Exercise and Nutrition Prehabilitation on Functional Capacity in Esophagogastric Cancer Surgery: A Randomized Clinical Trial. JAMA Surg 2018;153:1081-9.

17. Guinan EM, Forde C, O'Neill L, et al. Effect of preoperative inspiratory muscle training on physical functioning following esophagectomy. Dis Esophagus 2019;32:doy091.

18. Dettling DS, van der Schaaf M, Blom RL, et al. Feasibility and effectiveness of pre-operative inspiratory muscle 
training in patients undergoing oesophagectomy: a pilot study. Physiother Res Int 2013;18:16-26.

19. van Egmond MA, van der Schaaf M, Klinkenbijl JH, et al. Preoperative functional status is not associated with postoperative surgical complications in low risk patients undergoing esophagectomy. Dis Esophagus 2017;30:1-7.

20. van Egmond MA, van der Schaaf M, Klinkenbijl JHG, et al. The pre- and postoperative course of functional status in patients undergoing esophageal cancer surgery. Eur J Surg Oncol 2020;46:173-9.

21. Huang C, Wang Y, Li X, et al. Clinical features of patients

Cite this article as: Chen K, Yao F, Chen X, Lin Y, Kang M. Effectiveness of telerehabilitation on short-term quality of life of patients after esophageal cancer surgery during COVID-19: a single-center, randomized, controlled study. J Gastrointest Oncol 2021;12(4):1255-1264. doi: 10.21037/jgo-21-385 infected with 2019 novel coronavirus in Wuhan, China. Lancet 2020;395:497-506.

22. Kauppila JH, Xie S, Johar A, et al. Meta-analysis of healthrelated quality of life after minimally invasive versus open oesophagectomy for oesophageal cancer. Br J Surg 2017;104:1131-40.

23. Dai Z, Lang W, Yang H, et al. Validation of EORTC QLQ-OES18 for Chinese patients with esophageal cancer. Dis Esophagus 2017;30:1-7.

(English Language Editor: B. Draper) 


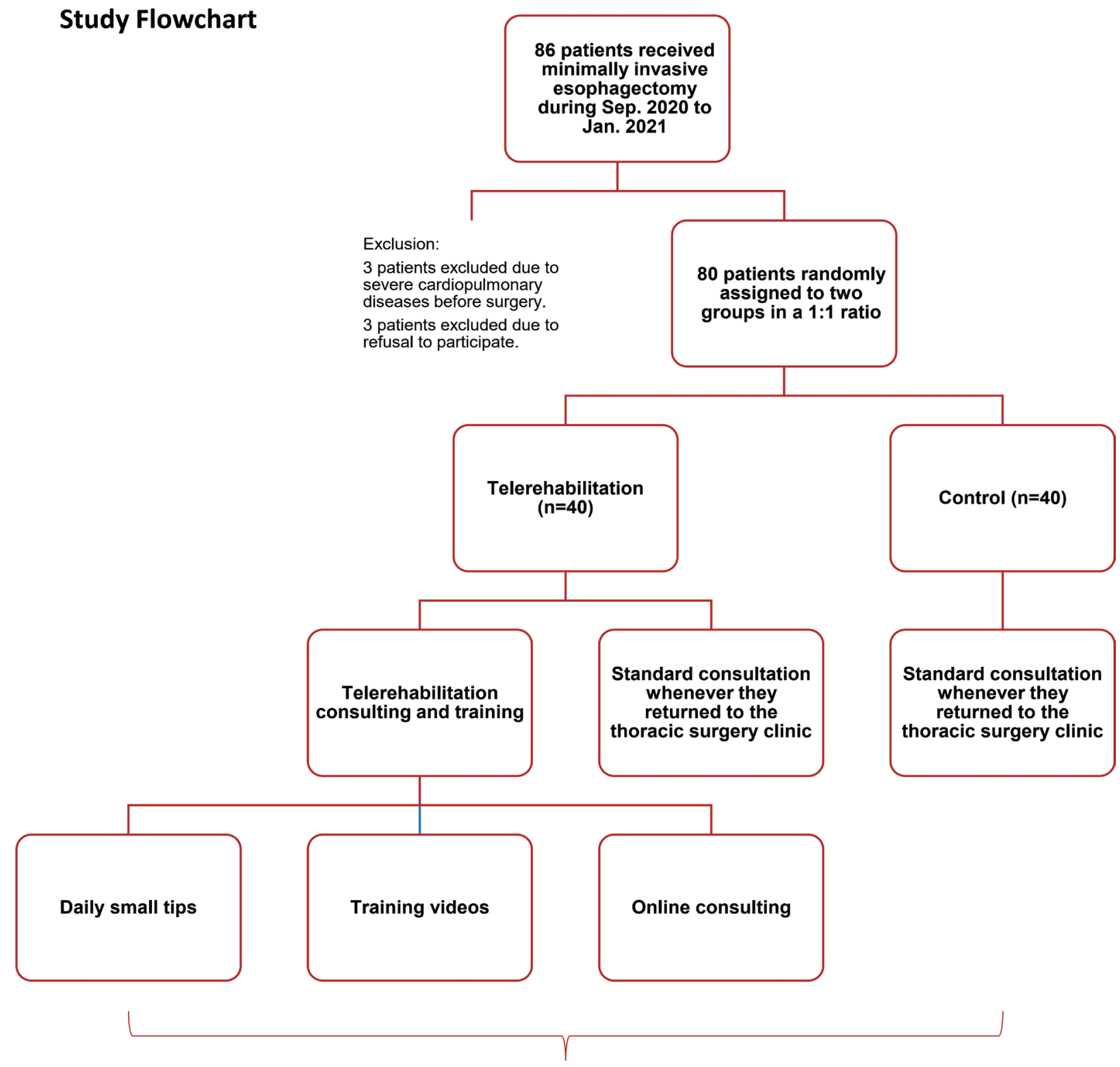

All 80 participants completed the 3-months follow-up

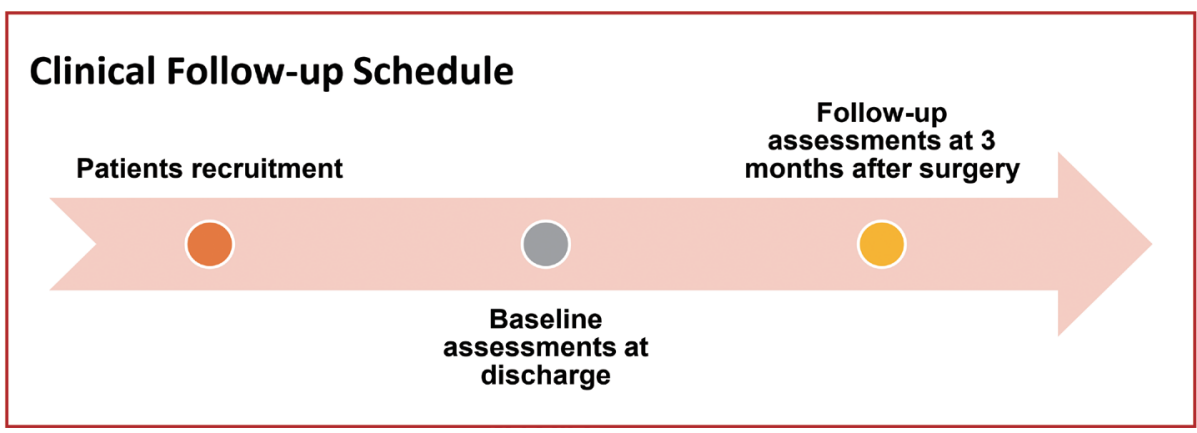

Figure S1 The study flowchart and clinical follow-up schedule. 